\title{
Philosophiques
}

\section{Jean-Pierre Cometti, Philosopher avec Wittgenstein, Paris, PUF (coll. « L'interrogation philosophique »), 1996, 246 p.}

\section{Pasquier Lambert}

Volume 25, numéro 2, automne 1998

Les modèles d'évolution en économie et en sciences sociales

URI : https://id.erudit.org/iderudit/027495ar

DOI : https://doi.org/10.7202/027495ar

Aller au sommaire du numéro

Éditeur(s)

Société de philosophie du Québec

ISSN

0316-2923 (imprimé)

1492-1391 (numérique)

Découvrir la revue

Citer ce compte rendu

Lambert, P. (1998). Compte rendu de [Jean-Pierre Cometti, Philosopher avec Wittgenstein, Paris, PUF (coll. «L'interrogation philosophique »), 1996, 246 p.] Philosophiques, 25(2), 294-296. https://doi.org/10.7202/027495ar d'utilisation que vous pouvez consulter en ligne.

https://apropos.erudit.org/fr/usagers/politique-dutilisation/ 


\section{Jean-Pierre Cometti, Philosopher avec Wittgenstein, Paris, PUF (coll. “L'interrogation philosophique »), 1996, 2/6 p.}

Après avoir écrit deux lives importants sur Robert Nusil PPLF, 1985; Nardaga, 1986, l'auteur s'intéresse maintenant à une autre figure autrichienne bien comue : Ludwig Wittgenstein. Ce live a été précédé de plusieurs articles sur l'esthéticque de Wingenstein et suivi d'un essai intitulé Lo masison de IVittgenstein (PUF, 1998), concernant la maison construite par IVittgenstein tout juste avant son retour à la philosophie en 1930 .

L'auteur, dans sa première publication entièrement consacré à Wiltgenstein, trate des rapports conflictuels qu'entretient celui-ci avec la philosophie el du difficile usage que l'on peut faire d'une pensée qui s'inserit à contre eourant de tout un mode. de philosopher propre a la eivilisation oceidentale chapitre premieri). It explicpue ensuite les notions de regle el de jen de langorge celiap. $\mathrm{I}$; puis celle de forme de sie (chap. III), sans nécessaircment apporter un éclairage nouveau sur ces notions, mais en metrant bien en perspeetive les liens qui les sous-tendent. L'auteur aborde ensuite un sujet qui semble lui tenir à cour : les questions esthétiques ichap. IV . Le livere se lemine sur la conception wittgenstemienne par excollence de la philosophie:

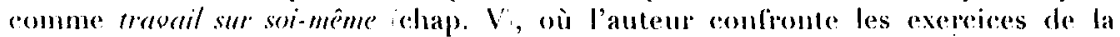
thérapeutique wittgensteiniemne avec les pratiques textuelles et la déconstruction.

Les prélentions anti-fondationnalistes et la vision anthropologique de Wittgenstein concernant les pratiques humaines telles que les rimels of les croyances aussi bien morales comme les rites religieux; que mathématiques la nécessité et les vérités éternelles, par exemplej sont bien rendues par l'auteur dans les chapitres II et III, quoiqu'il ait parfois du mal à tenir une ligne d'argumentation linéaire; bref, il prend de nombreux détours pour expliquer certaines réflexions wittgensteiniennes bien connues et relativement faciles à résumer. L'auteur prétend que règles, jeu de langruge et forme de vie sont des notions qui n'ont pas de prétention fondationnelle, car Wittgenstein ne cherche pas à s'en servir comme base d'un systène ou d'une théorie, comme c'est le cas chez Habermas avec sa "thécrie de l'agir communicationnel ". Par conséquent, elles n'ont pas non plus de portée directement sociale ou politique. Elles sont plutôt de nouvelles métaphores pour explicpuer le fonctionnement du langage dans un contexte philosophique. Nais puisque le langage remplit certaines fonctions qui ne sont pas étrangères à ses dimensions sociale et politique, touchant la culture et la communication, J.-P. Comelti eroit bon de confronter les vues de Willgenstein à la "théorie de la discussion n de Apel et Ilabermas de mêne qu'a l'interprétation de Kripke, allant jusqu'à affirmer que "Kripke rejoint à sa manière Apel et Habermas par son souci d'une justification qui ne soit pas seulement grammaticale et interne à nos jeux de langage, et par une conception dont les bases paraissent étroitement consensuelles et très peu ancrées dans des formes de vie n $(p .96$. Là où l'on doit donner raison à l'auteur, c'est que la vision anthropologique - el même anthropomorphique - de Wittgenstein concernant les pratiques humaines, vient de sa volonté de justifier les pratiques sans " sortir du langage ", simplement parce qu'elles existent, parce qu'elles sont d'usage au sein d'une "forme de vie " partagée par une communauté, ce qui n'est pas le cas d'une grande majorité de philosophes, qui semblent croire à la nécessité de dégager le "vrai "fondement de nos pratiques. J. Bouveresse et J.-P. Cometti à sa suite n'ont donc pas tort de qualifier Wittgenstein de conventionnaliste, par opposition aux " consensualistes ".

Le chapitre IV est consacré à l'examen du geste et de l'aspect en esthétique. L'auteur en profite aussi pour nous resservir la distinction wittgensteinienne bien comnue entre les causes et les raisons. Le nœud de la discussion est que l'appréciation esthétique n'est pas une réaction causale, de nature psychologique, à une ceuvre d'art, 
quoiqu'une œuvre d'art puisse être la raison d'un certain type de réaction émotive. Il s'agit plutôt de saisir certains aspects de l'œuvre comme représentant ou suggérant telle ou telle chose. Le rôle du geste, en art, est précisément de montrer un aspect de l'cuvre (picturale ou musicale) qui n'avait pas été saisi auparavant. C'est pourquoi, comme le dit si bien Wittgenstein, a il est difficile en art de dire quelque chose d'aussi bon que... ne rien dire "Vermischte Bemerkungen, p. 33).

L'intérêt principal de ce livre, cependant, réside surtout dans les premier et dernier chapitres, où sont soulevées les questions de l'importance, de l'intérêt et de la pertinence de la thérapeutique de Wittgenstein. Le projet de Wittgenstein, cela est bien connu, est de guérir l'entendement des philosophes des illusions causées par un usage fautif du langage. La philosophie, façonnée par le langage, ne contiendrait essentiellement que des problèmes "grammaticanx " que seule une vision sunoptique serail en mesnre de dissoudre. Celte orientation thérapeuticque fait en sorto que les positions habituedlement défendues et débattues en philosophie ne se retrouvent pas dans l'ceuvre de Wiłtgenstein, sinon pour être critiquées.

Ainsi, "le probleme que pose Wittgenstein aux philosophes reste celui cu'il pose à la philosophie " p. 216\%. C'est dire qu'il est question ici de s'interroger sur le sens même de l'activité philosophique. Cela nous oblige à nous demander ce cu'on peut faire d'une critique négative comme celle de Wittgenstein, qui se refuse à construire quelque chose de positif pour, au contraire, remettre en question la légitimité même de l'activité philosophique : "Une philosophie qui ne se donne pas d'autre tâche que de guérir ou de dissiper les malaises auxquels certains usages linguistiques donnent lieu doit nécessairement à ces mêmes usages sa raison d'exister, au point que si, d'aventure, son action thérapeutique devait aboutir, elle lui serait d'une certaine manière fatale " p. 216;. N'est-ce pas pour cela que Wittgenstein s'interroge sur la raison d'être de son activité philosophiçue en se demandant : "Est-ce que je souhaite voir mon travail continué par d'autres plutôt qu'un changement dans la manière de vive qui rende loutes ces questions superflues, cela n'est pas du tout clair pour moi ic'est pourquoi je ne pourrai jamais fonder une école of lemische Bemerkungen, p. 7/, ?' Cela lait de la philosophie une activité purement contingente, de sorte que " nous devons pouvoir eesser de philosopher lorsque nous lo voulons n Philosophische Lmersuchungen, \$133. Tel est le paradoxo de la deuxième philosophie de Witlgenstein, dont Wittgenstein lui-même s'était d'aillems aperçu le prenier.

Dans la veine des eritiques de Hacker et Baker, Cometti constate également qu'il y a d' "authentiques penseurs", comme Wittgenstein, "à côlé des déconstructionuistes el des philosophes analytiques oceupés à leurs jeux oisil's " (sic) (j. 18), citant Putnam, discutant les thèses de Quine, Davidson ef Goodman, consactant une section importante à examiner l'inlerprétation controversée de kripke, de même qu'une autre à opposer Witgenstein et Derrida. On peut se demander jusqu'à quel point cette manière de "philosopher aves: Wintgenstein o contre la tradition est une interprétation plus " autbentique". Eist-il raiment plus léggitime d'utiliser W'ittgenstein contre la philosophe analytique el la cléconstruetion, comme le font Hacker, Baker et Conneti, yue de l'utiliser pour y puiser un certain nombre de theses cléfendues moins par Willgenstein que par la philosophie analyticue en général ? On peut présumer que Wittgenstein se setait défendu d'appartenir à une queleonque école ou tendance et aurait tout simplement refusé que ses idlées soient utilisées à cles lins polémiques.

D'autre part, il est regrettable que cette monographie soit aflligée, quoique à un moindre degré, du même défaut que la plupart des longs ouvrages consacrés à Wittgenstein en français : l'auteur saute souvent d'un sujet à un autre, opérant des transitions thématiques sans se soucier de systématiser les interprétations successives qu'il donne des clivers fragments de l'cuvre de Wittgenstein et citant au passage des auteurs aussi différents que peuvent l'être Apel, Habermas, Kant, Heidegger, Rorty ou 
Musil, en plus de ceux mentionnés précédemment. Les chapitres II et III souffrent particulièrement de leur caractère rapsodique. Quant au chapitre IV, le thème de l'esthétique sort un peu du cadre général de l'ouvrage.

Certains protesteront qu'agir autrement ne ferait que muire à l'exposition fidele des pensées de Wittgenstein, ce dernier (rlu moins dans sa deuxième période récusant toute explieation de nature théorique ou systematique en philosophie. En elfet, des que l'on lente de saisir la pensée de IVittgenstein de laçon șistématique, on risque de

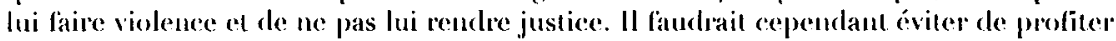
des philosophies qui se présentent sous forme de remargues ou de paragraphes brefs, comme celles de Nietzsehe et Wiltgenstein, de manière à en tirer les interprétations les plus diverses et les plus contradictoires ou à les utiliser eomme cheval de bataille contre les autres philosophies, ce qui n'est pas leur rende service non plus. Ces difficultess, par contre, en disent déjà beaucoup sur la nature des entreprises philosophiques anti-théoriques et non systématiques, ainsi que sur les problèmes exégétiques qu'elles posent; d'où, encore une fois, la difficulté de a philosopher aver: Wittgenstein".

Par son livre, qui s'impose comme une contribution importante aux éludes wittgensteiniennes, J.P. Conetti met bien en lumière l'ambiguïté du projet de Wittgenstein durant sa seconde période, au point où l'on se demande si le probleme que Wittgeustein pose à la philosophie colui de sa légitimité n'est pas au fond le problème qu'il éprouve face à la philosophice. (In pourait dès lor's reprocher à Wittgenstein de voir la philosophie comme essenticllement problématique, alors que c'est lui-même, en raison de son attitude personnelle concemant la philosophic, qui rend l'activité philosophique problématique.

Pasquier Lambert

Université de Nontréal 